\title{
Auction-Based Resource Allocation for Hierarchical Wireless Mesh Networks in Rural Areas
}

\author{
Zhen Kong \\ Electrical and Computer Engineering \\ Colorado State University \\ Fort Collins, CO, USA \\ zhen.kong@colostate.edu
}

\author{
Yu-Kwong Kwok \\ Electrical and Electronics Engineering \\ The University of Hong Kong \\ Pokfulam, Hong Kong \\ ykwok@hku.hk
}

\begin{abstract}
Hierarchical wireless mesh networks (WMNs), in which multiple layers of mesh routers (MRs) share one centralized gateway, are gaining importance in providing broadband Internet services in rural areas. Such WMNs are effective in that they can provide a wide coverage area in a cost effective manner. However, more often than not, such MRs are owned and operated by different autonomous entities, which might not have the incentive to comply with a central rule. Thus, in such a competitive non-cooperative environment, effective resource allocation is a notoriously difficult problem. In this paper, we propose a novel auction based framework, in which the gateway, equipped with the precious high speed Internet connection, serves as the auctioneer, while the first-level MRs (i.e., those with direct wireless connections to the gateway) act as bidders competing resources among each other. Specifically, a major novelty of our framework is that the valuation and payment functions are derived based on practical considerations and have realistic physical significance. Based on this framework, we first present the Vickrey-Clarke-Groves (VCG) based auction approaches. To further improve performance, we then propose two novel relay auction (RA) algorithms and analyze the effectiveness of their bidding strategies using the game theoretic solution concept of Nash equilibrium (NE). To realize the NE in a practical distributed computing fashion, we also design an iterative bid updating algorithm. Through simulations, we find that the proposed RA algorithms can achieve competitive performance in terms of resource allocation efficiency as the VCG ones, while giving much better connection blocking probability performance, and also having the capability to balance the efficiency and fairness by adjusting the payment function.
\end{abstract}

\section{Categories and Subject Descriptors}

C.2.1 [Network Architecture and Design]: Wireless communication; C.4 [Performance of Systems]: Performance attributes

Permission to make digital or hard copies of all or part of this work for personal or classroom use is granted without fee provided that copies are not made or distributed for profit or commercial advantage and that copies bear this notice and the full citation on the first page. To copy otherwise, to republish, to post on servers or to redistribute to lists, requires prior specific permission and/or a fee.

CHANTS'09, September 25, 2009, Beijing, China.

Copyright 2009 ACM 978-1-60558-741-7/09/09 ...\$10.00.

\section{General Terms}

Algorithms, Design, Performance

\section{Keywords}

Wireless mesh networks, auction algorithms, resource allocation, IEEE 802.16

\section{INTRODUCTION}

Wireless mesh networks (WMNs) [1] are widely envisioned to be a key technology to improve the capacity and coverage for wireless broadband access services at affordable costs in rural areas where wired communication infrastructure is too costly to install. A typical WMN consists of mesh routers (MRs) that not only provide wireless access for mesh clients (MCs) but also form the backbone of the network. To provide broadband out-bound access, gateways are usually installed between the mesh backbone and the Internet. Consequently, as shown in Fig. 1, to provide broadband Internet services to a remote area, the MRs close to the gateway can work as relay nodes transmitting traffic between the Internet and the MRs far away from the gateway. Among contemporary wireless technologies, IEEE 802.16 standard has been considered as a promising solution for WMNs due to its capacity to combat channel fading and support high data rate, and the research and deployment of IEEE 802.16-based WMNs have gained enormous popularity recently [14].

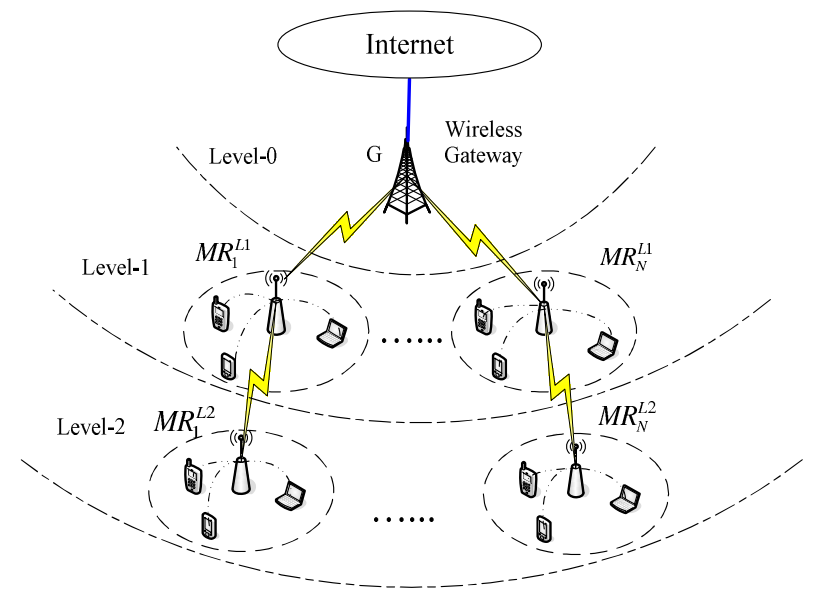

Figure 1: Structure of a hierarchical wireless mesh network. 
One of the key enabling features behind such a hierarchical WMN is that a MR should not only serve the MCs in its own local cell, but also relay traffic for outer level MRs. Unfortunately, this kind of cooperation among MRs is not always practical in reality, especially when these MRs are owned by different profit-maximizing entities so that they have to compete with each other for radio resource. In such a non-cooperative environment, a MR will rationally exhibit selfish behaviors driven by self-interests in that the MR may report a bogus channel information or valuation so as to improve its own benefit [11], or refuse to relay other MRs' traffic because relaying traffic will inevitably consume its own resource and consequently degrade the performance of its local cell. Though these non-cooperative behaviors could improve the performance for the selfish MR itself, they may lead to inefficient or unfair resource utilization for the whole system.

Though IEEE $802.16 \mathrm{j}[8]$ is aimed at infrastructure meshing, it is still under discussion and many components are not complete yet [17]. Several recent works have addressed the problem of resource allocation in WMNs. In [16], a radio resource management framework for scheduling and admission control in an IEEE 802.16-based WMN was proposed to maximize transmission rate as well as satisfy QoS requirements under power constraint in a wireless MR. Unfortunately, it just provided a study of the performance of an individual router; overall system performance was not studied. In [3], a joint power-frequency-time resource allocation algorithm was proposed to optimize the system performance of a clustered WMN. This approach was based on global optimization, while the competition among different MRs was not considered. In [18], the throughput maximization problems in a WMN for both cooperative and non-cooperative scenarios were analyzed, and a linear pricing scheme was proposed to combat the selfish behavior of non-cooperative MRs. However, the strategy to relay traffic was not considered. In [15], a bandwidth allocation scheme based on a bargaining game formulation was proposed to allocate resource fairly in an integrated WLAN/WMAN multihop mesh network. Yet this model is based on the situation where the players cooperatively bargain with each other to make an agreement, which may not be realistic in a non-cooperative environment. In [7], two auction-based approaches were proposed to help relay nodes to determine how to allocate transmission power among users as well as improve fairness and efficiency performances. Unfortunately, in their model a relay node itself does not have local users to serve, which is different with our model where a relay MR should serve both local and relay connections.

The resource allocation problem in a competitive environment can be effectively addressed by means of auction theory [12], which is widely known to be efficient in allocating resources in a non-cooperative situation, and is the focus of our study. Specifically, in this hierarchical mesh infrastructure, we assume using an IEEE 802.16 WirelessMAN-OFDM air interface, where the physical layer is based on orthogonal frequency-division multiplexing (OFDM), and the media access control (MAC) scheme is based on time-division multiple access (TDMA). With OFDM/TDMA, all subchannels are allocated to one connection at a time. Thus, in our model, the auction goods are time-slots, the auctioneer is the gateway who has radio resource, i.e., time-slots, to allocate, and the bidders are MRs who need to request time-slots from the gateway and use them for local or relay traffic transmissions.

To implement an auction approach in WMNs, two challenging problems must be tackled. First, a succinct and expressive bidding language is necessary. Here, we define a bidder's valuation function by quantifying its valuation on the allocated resource under the current state, and then fully characterize it simply by a single scalar parameter. Thus, each bidder can submit the scalar as its bid, thereby leading to an efficient and practical bidding process. Second, how should we define "payment"? In some auction-based algorithms [7, 19], payments are expressed in terms of money or some kinds of virtual parameters, and thus, suffer from a lack of practical meanings and significance. Here we consider associating payment with the time-slots used by an MR to relay other MR's packets. Specifically, to win time-slots allocated by the gateway, a bidder must "pay" some wireless resources, i.e., time-slots, and use them to forward an outer level MR's traffic.

Our contributions are as follows. We first define a linear valuation function based on time-slots. Consequently, a bidder's utility is just the value it receives minus the assigned payment. The auction approach based on VCG auction [12] can then be used to solve this model with linear utility [2]. On the other hand, we find that a concave valuation function is more suitable to represent bidder's valuation and the corresponding utility because it can represent the saturation of user QoS satisfaction as the received resource increases. Thus, we further define a family of concave valuation and utility functions, and derive a Quasi-VCG (QVCG) method, which employs the VCG allocation and payment policies under this non-linear environment. Unfortunately, there is one severe drawback in VCG-based algorithms in that they do not consider fairness performance [2]. This is clearly undesirable in a wireless resource allocation environment where the gateway should provide some level of QoS guarantees in terms of fairness. To overcome the drawback of the VCG algorithms, we further propose a novel relay auction (RA) framework based on a concave utility function, where the bid submitted by a MR is a scalar associated with its reported valuation, while the payment is increasing with this bidding scalar value. Specifically, depending on the type of payment function, i.e., linear or logarithmic payment function, we design two RA algorithms, called RALiP and RALoP. We first prove the existence and uniqueness of Nash equilibrium (NE) [5]. To realize the NE in a practical distributed computing fashion, we propose an iterative bid updating algorithm. We then compare the performance of these auction algorithms through simulations, and find that the proposed RA algorithms can achieve aggregate throughput performance comparable to VCG approach, while they can adjust the resource ratio allocated to different level of MRs, so as to strike a proper balance between efficiency and fairness. From a service dependability perspective, the performance of our RA algorithms is also better than VCG in terms of connection blocking probability. We also show the convergence of bids to NE for our proposed RA methods. Finally, the performance of some traditional cooperative resource allocation algorithms are also analyzed in this non-cooperative environment, and we find that they are not suitable in a non-cooperative situation when compared with the proposed auction algorithms.

The rest of this paper is organized as follows. We present 
the system model and auction model in Section 2. Section 3 presents and analyzes VCG-based auction algorithms. In Section 4, our novel relay auction approaches are proposed. Section 5 gives the simulation results. We conclude in Section 6 .

\section{MODEL}

\subsection{System Model}

In this paper, we consider an IEEE 802.16 OFDM/TDMA based WMN with three hierarchical levels as shown in Fig. 1 and focus on downlink resource allocation. Such a hierarchical network structure is highly practical and can be used to model an infrastructure WMN to serve the outskirts of a small town in rural areas. At Level-0, there is a wireless gateway $G$ that has direct connection to the Internet. There are $N$ Level-1 MRs, denoted by $M R_{i}^{L_{1}}, i \in \mathbb{N}=\{1, \ldots, N\}$, surrounding the gateway and providing wireless access for their clients. Each MR and its corresponding MCs form a cell. Outside Level-1, to cover a wide geographical area (a necessary condition in a rural area), there are $N$ other MRs, denoted by $M R_{i}^{L_{2}}$, which are located far from $G$. As a result, each $M R_{i}^{L_{2}}$ does not have direct wireless connections with the gateway, and it can only get broadband Internet services through the relaying services provided by $M R_{i}^{L_{1}}$. We also assume that $M R_{i}^{L_{1}}$ in Level-1 just relays traffics to $M R_{i}^{L_{2}}$ in Level-2. Every Level-1 MR competes with each other for the bandwidth resource provided by the gateway.

We assume all cells operate under IEEE 802.16 OFDM/TD MA-TDD mode, and each adjacent cell uses a different frequency band. The MAC frame is composed of downlink and uplink subframes. Each downlink subframe is composed of $T_{d}$ time-slots, each of which is used for transmission of packets corresponding to one connection. For uplink and downlink transmission using OFDM, each MR uses $M$ subchannels with total bandwidth of $B \mathrm{MHz}$. There are $R_{i}^{G L_{1}}$ packets per time-slot transmitted from the gateway to $M R_{i}^{L_{1}}$. We use $P E R_{i}^{L_{1}}$ and $P E R_{i}^{L_{2}}$ to denote the average packet error rates in the downlink of $M R_{i}^{L_{1}}$ 's and $M R_{i}^{L_{2}}$ 's local cell, respectively. These PERs depend on the PHY layer packet transmission error rate and MAC layer packet dropping rate. Then we can get the average number of packets that can be transmitted successfully per time-slot from $M R_{i}^{L_{1}}$ and $M R_{i}^{L_{2}}$ as $a_{i}^{L_{1}}$ and $a_{i}^{L_{2}}$, respectively, i.e.,

$$
\begin{gathered}
a_{i}^{L_{1}}=R_{i}^{G L_{1}} \cdot\left(1-P E R_{i}^{L_{1}}\right) \\
a_{i}^{L_{2}}=a_{i}^{L_{1}} \cdot\left(1-P E R_{i}^{L_{2}}\right)
\end{gathered}
$$

We also assume that there are $N_{i}^{L_{1}}$ MCs served by $M R_{i}^{L_{1}}$, and $N_{i}^{L_{2}}$ MCs served by $M R_{i}^{L_{2}}$. Thus, when $T_{i}^{L_{1}}$ time-slots are allocated to $M R_{i}^{L_{1}}$ for its local usage and $T_{i}^{L_{2}}$ time-slots are used to relay Level-2 MR's traffic, the average received packets per frame by a Level-1 MC and a Level-2 MC are then given by:

$$
\begin{aligned}
& \bar{Q}_{i}^{L_{1}}\left(T_{i}^{L_{1}}\right)=\frac{a_{i}^{L_{1}}}{N_{i}^{L_{1}}} \cdot T_{i}^{L_{1}} \\
& \bar{Q}_{i}^{L_{2}}\left(T_{i}^{L_{2}}\right)=\frac{a_{i}^{L_{2}}}{N_{i}^{L_{2}}} \cdot T_{i}^{L_{2}}
\end{aligned}
$$

\subsection{Auction Framework}

In our framework, each $M R_{i}^{L_{1}}$ needs to compete for timeslots through auction, and the valuation function can be fully characterized by a scalar valuation parameter. Specifically, every $M R_{i}^{L_{1}}$ calculates the valuation parameter $a_{i}^{L_{1}}$ and then submits a bid $b_{i}$ to the gateway according to a rationally selfish strategy. For $M R_{i}^{L_{2}}$, because $a_{i}^{L_{2}}$ depends on $P E R_{i}^{L_{2}}$ and $M R_{i}^{L_{1}}$ 's reported valuation parameter, we assume that every $M R_{i}^{L_{2}}$ can send $P E R_{i}^{L_{2}}$ to the gateway via a secure out-of-band control channel through $M R_{i}^{L_{1}}$. After receiving the announced bidding profile $B=\left\{b_{1}, b_{2}, \ldots, b_{N}\right\}$ from every $M R_{i}^{L_{1}}$, and all level-2 $P E R_{i}^{L_{2}}$, the gateway will know each MR's reported valuation, and then calculate the allocation $T=T(B)=\left\{T_{1}, T_{2}, \ldots, T_{i}, \ldots, T_{N}\right\}$, which represents the number of time-slots allocated to each $M R_{i}^{L_{1}}$, as well as the payment $P=P(B)=\left\{T_{1}^{L_{2}}, T_{2}^{L_{2}}, \ldots, T_{i}^{L_{2}}, \ldots, T_{N}^{L_{2}}\right\}$, which represents the number of time-slots that should be used by $M R_{i}^{L_{1}}$ to relay $M R_{i}^{L_{2}}$ 's traffic. Subsequently, the allocation and payment results are transmitted to each $M R_{i}^{L_{1}}$. Upon receiving them, each $M R_{i}^{L_{1}}$ gets to know $T_{i}^{L_{1}}=$ $T_{i}-T_{i}^{L_{2}}$ and payment $T_{i}^{L_{2}}$.

In a typical auction, the utility of a bidder is the value received by this bidder minus the payment assigned by the auctioneer. However, in our model, to provide practical meanings for payment, we associate the payment with the practical radio resource, i.e., time-slots. Thus, when $M R_{i}^{L_{1}}$ receives $T_{i}$ time-slots and accept $T_{i}^{L_{2}}$ time-slots as payment, its ultimate utility is its true valuation when getting $T_{i}^{L_{1}}=T_{i}-T_{i}^{L_{2}}$, i.e.,

$$
U_{i}^{L_{1}}\left(T_{i}, T_{i}^{L_{2}}\right)=V_{i}^{L_{1}}\left(T_{i}-T_{i}^{L_{2}}\right)=V_{i}^{L_{1}}\left(T_{i}^{L_{1}}\right)
$$

For $M R_{i}^{L_{2}}$, its utility is just its valuation with $T_{i}^{L_{2}}$ timeslots, i.e.,

$$
U_{i}^{L_{2}}\left(T_{i}^{L_{2}}\right)=V_{i}^{L_{2}}\left(T_{i}^{L_{2}}\right)
$$

\section{VCG AUCTION}

\subsection{Classical VCG Based on a Linear Utility Function}

Vickrey-Clarke-Groves (VCG) auction has been regarded as one of the most effective mechanisms to induce truthrevealing strategies [2], i.e., every bidder truthfully declares their resource requirements, and to maximize the declared social welfare, i.e., aggregate valuation of all bidders, by charging each bidder a payment corresponding to the inconvenience it causes to other bidders. The VCG theory relies on the restrictive assumption that bidders' utility functions are quasi-linear, i.e., the utility can be expressed as the value of the goods received minus the payment made. To fulfill this requirement, we first design a VCG auction-based algorithm using a linear utility function, called a Classical-VCG (CVCG) algorithm, where the valuation function is given by the maximal feasible received packets per time-slot as discussed in Section 1, i.e.,

$$
\begin{aligned}
& V_{i}^{L_{1}}\left(T_{i}^{L_{1}}\right)=\bar{Q}_{i}^{L_{1}}\left(T_{i}^{L_{1}}\right) \cdot N_{i}^{L_{1}}=a_{i}^{L_{1}} \cdot T_{i}^{L_{1}} \\
& V_{i}^{L_{2}}\left(T_{i}^{L_{2}}\right)=\bar{Q}_{i}^{L_{2}}\left(T_{i}^{L_{2}}\right) \cdot N_{i}^{L_{2}}=a_{i}^{L_{2}} \cdot T_{i}^{L_{2}}
\end{aligned}
$$

Thus, their utilities can be expressed as:

$$
U_{i}^{L_{1}}\left(T_{i}, T_{i}^{L_{2}}\right)=a_{i}^{L_{1}} \cdot T_{i}-a_{i}^{L_{1}} \cdot T_{i}^{L_{2}}
$$




$$
U_{i}^{L_{2}}\left(T_{i}^{L_{2}}\right)=a_{i}^{L_{2}} \cdot T_{i}^{L_{2}}
$$

Notice that (7) is strictly increasing on $a_{i}^{L_{1}}$. Thus, to maximize the declared aggregate valuation, the auctioneer just needs to allocate all time-slots to the bidder with the highest $a_{i}^{L_{1}}$. Then, the sealed-bid second-price auction [12], which is a special case of VCG auction, can be used to simplify the process of calculating allocation and payment results. Based on the above linear model, we design an auction mechanism based on the classic VCG theory as follows.

1. Each bidder $M R_{i}^{L_{1}}$ submits its true valuation parameter $a_{i}^{L_{1}}$ as bid $b_{i}$ to the gateway, i.e., $b_{i}=\mu_{i}\left(a_{i}^{L_{1}}\right)=$ $a_{i}^{L_{1}}$.

2. The gateway chooses the bidder $i^{*}$ with the highest reported valuation to get all time-slots, i.e.,

$$
i^{*}=\arg \max _{i}\left(b_{i}\right)
$$

3. To determine the payment, the gateway first chooses the second highest valuation $\max _{j \neq i *}\left(b_{j}\right)$, which is then converted to the number of time-slots used by $M R_{i}^{L_{1}}$ to forward the traffic to $M R_{i}^{L_{2}}$, i.e.,

$$
T_{i^{*}}^{L_{2}}=\frac{\max _{j \neq i^{*}}\left(b_{j}\right) \cdot T_{d}}{a_{i^{*}}^{L_{2}}}
$$

\subsection{Quasi-VCG Based on a Concave Utility Func- tion}

While the above CVCG algorithm based on classical VCG model is very easy to implement, the linear utility function has a drawback in that it cannot capture the "diminishing return" effects in satisfying the QoS requirements of wireless service users. Specifically, we consider the following logarithmic function to express a level-1 MC's valuation on its average received packets per frame, i.e.,

$$
v_{i}^{L_{1}}\left(a_{i}^{L_{1}}, T_{i}^{L_{1}}\right)=\log \left(\bar{Q}_{i}^{L_{1}}\left(T_{i}^{L_{1}}\right)+1\right)=\log \left(\frac{a_{i}^{L_{1}}}{N_{i}} \cdot T_{i}^{L_{1}}+1\right)
$$

Thus, when there are $N_{i}^{L_{1}}$ MCs served by $M R_{i}^{L_{1}}$, its valuation on $T_{i}^{L_{1}}$ time-slots can be defined as:

$V_{i}^{L_{1}}\left(a_{i}^{L_{1}}, T_{i}^{L_{1}}\right)=N_{i}^{L_{1}} \cdot v_{i}^{L_{1}}\left(a_{i}^{1}, T_{i}^{L_{1}}\right)=N_{i}^{L_{1}} \cdot \log \left(\frac{a_{i}^{L_{1}}}{N_{i}^{L_{1}}} \cdot T_{i}^{L_{1}}+1\right)$

Similarly, $M R_{i}^{L_{2}}$ 's valuation on $T_{i}^{L_{2}}$ time-slots is:

$$
V_{i}^{L_{2}}\left(a_{i}^{L_{2}}, T_{i}^{L_{2}}\right)=N_{i}^{L_{2}} \cdot \log \left(\frac{a_{i}^{L_{2}}}{N_{i}^{L_{2}}} \cdot T_{i}^{L_{2}}+1\right)
$$

Thus, according to (9) and (10), their utilities are:

$$
\begin{gathered}
U_{i}^{L_{1}}\left(T_{i}, T_{i}^{L_{2}}\right)=V_{i}^{L_{1}}\left(a_{i}^{L_{1}}, T_{i}-T_{i}^{L_{2}}\right) \\
U_{i}^{L_{2}}\left(T_{i}^{L_{2}}\right)=V_{i}^{L_{2}}\left(a_{i}^{L_{2}}, T_{i}^{L_{2}}\right)
\end{gathered}
$$

Obviously, $U_{i}^{L_{1}}$ is concave on both valuation parameter $a_{i}^{L_{1}}$ and allocated resource $T_{i}^{L_{1}}$, while $U_{i}^{L_{2}}$ is also concave on both $a_{i}^{L_{2}}$ and $T_{i}^{L_{2}}$. These utility functions are not quasilinear, implying that they cannot be simply expressed as the difference between the valuation and payment. Thus, the classical VCG method cannot be applied directly. In the following, based on the concave utility function in a non-linear setting, we propose a novel auction approach employing the VCG allocation and payment policies.

1. Each $M R_{i}^{L_{1}}$ submits its bid $b_{i}=a_{i}^{L_{1}}$ to the gateway.

2. The gateway computes an outcome that maximizes the declared social welfare, i.e.,

$$
\begin{aligned}
T_{*} & =\left\{T_{1}^{*}, \cdots, T_{N}^{*}\right\}=\arg \max _{\left\{T_{i}\right\}} \sum_{i} V_{i}^{L_{1}}\left(a_{i}^{L_{1}}, T_{i}\right) \\
& =\arg \max _{\left\{T_{i}\right\}} \sum_{i} N_{i}^{L_{1}} \cdot \log \left(\frac{a_{i}^{L_{1}}}{N_{i}^{L_{1}}} \cdot T_{i}+1\right)
\end{aligned}
$$

subject to:

$$
\text { (I) } \sum_{i} T_{i}=T_{d} \quad(I I) 0 \leq T_{i} \leq T_{d}
$$

3. To determine the payment, the gateway first calculates the loss of social welfare due to $M R_{i}^{L_{1}}$ 's presence, i.e.,

$$
P_{i}=\max _{i} \sum_{j \neq i} V_{j}\left(T_{j}\right)-\sum_{j \neq i} V_{j}\left(T_{j}^{*}\right)
$$

$P_{i}$ is further converted to the number of time-slots $T_{i}^{L_{2}}$ used by $M R_{i}^{L_{1}}$ to relay the traffic to $M R_{i}^{L_{2}}$, i.e.,

$$
T_{i}^{L_{2}}=\left(V_{i}^{L_{2}}\right)^{-1}\left(P_{i}\right)
$$

Here, our proposed auction mechanism applies the VCG allocation and payment policies to a non-linear environment, and thus we refer it to as Quasi-VCG (QVCG) algorithm.

There is one severe drawback in VCG-based mechanism in that both CVCG and QVCG approaches do not consider fairness performance [2]. This is clearly undesirable in a wireless resource allocation environment where the gateway should provide some level of QoS guarantees in terms of fairness. Thus, in the following section, we propose another new auction approach.

\section{RELAY AUCTION}

Generally, the more resource a bidder requests, the higher payment it should pay to the auctioneer. Thus, we define the payment as a function increasing with $b_{i}$, and propose a relay auction (RA) algorithm, which is formalized as follows.

\subsection{Auctioning Mechanism}

1. Bidding rule: $M R_{i}^{L_{1}}$ submits a scalar value $b_{i}$ to the gateway, and let $\mathbf{b}=\left\{b_{i}: b_{\min } \leq b_{i} \leq b_{\max }\right\}$. We also let $M R_{i}^{L_{2}}$ send its average packet error rate $P E R_{i}^{L_{2}}$ to the gateway via a secure out-of-band control channel through $M R_{i}^{L_{1}}$. Then, the gateway can know $M R_{i}^{L_{2}}$ 's valuation as $a_{i}^{L_{2}}=b_{i} \cdot\left(1-P E R_{i}^{L_{2}}\right)$.

2. Allocation rule:

$$
\begin{aligned}
T^{*} & =\left\{T_{1}^{*}, T_{2}^{*}, \cdots, T_{N}^{*}\right\}=\arg \max _{\left\{T_{i}\right\}} \sum_{i} U_{i}^{L_{1}}\left(T_{i}\right) \\
& =\arg \max _{\left\{T_{i}\right\}} \sum_{i}\left(N_{i}^{L_{1}} \cdot \log \left(\frac{b_{i}}{N_{i}^{L_{1}}} \cdot\left(T_{i}-T_{i}^{L_{2}}\right)+1\right)\right. \\
& \left.+N_{i}^{L_{2}} \cdot \log \left(\frac{b_{i} \cdot\left(1-P E R_{i}^{L_{2}}\right)}{N_{i}^{L_{2}}} \cdot T_{i}^{L_{2}}+1\right)\right)
\end{aligned}
$$


subject to:

$$
\text { (I) } \sum_{i}\left(T_{i}^{L_{1}}+T_{i}^{L_{2}}\right)=T_{d} \quad(I I) T_{i}^{L_{2}}=f\left(b_{i}\right) \cdot T_{i}
$$

3. Payment rule: If $M R_{i}^{L_{1}}$ gets $T_{i}$ time-slots, it must use $T_{i}^{L_{2}}=f\left(b_{i}\right) \cdot T_{i}$ time-slots for relaying $M R_{i}^{L_{2}}$ 's traffic, where $f\left(b_{i}\right)$ is increasing with $b_{i}$, and $0 \leq f\left(b_{i}\right) \leq$ 1. Here, we consider two different payment functions: $f_{1}\left(b_{i}\right)=\frac{b_{i}}{A}$, and $f_{2}\left(b_{i}\right)=\frac{\log \left(b_{i}+1\right)}{A}$, where $A$ is a payment parameter.

\subsection{Nash Equilibrium}

Our proposed relay auction can be formulated as a strategic non-cooperative game, i.e., $\Gamma \triangleq\left[\mathbb{N},\left\{b_{i}\right\}_{i \in \mathbb{N}},\left\{U_{i}^{L_{1}}(\mathbf{B})\right\}_{i \in \mathbb{N}}\right]$, with $N$ players, where $\mathbb{N} \triangleq\{1, \cdots, N\}$ is the player set, $\left\{b_{1}, \cdots, b_{N}\right\}$ is the action profile, and $U_{i}^{L_{1}}$ is the utility function of player $i$.

A useful solution concept of such a game is called a Nash equilibrium (NE) [5], which is a bidding profile $B^{*}$ where no MR wants to deviate unilaterally, i.e.,

$$
U_{i}^{L_{1}}\left(b_{i}^{*} ; \mathbf{b}_{-i}^{*}\right) \geq U_{i}^{L_{1}}\left(b_{i} ; \mathbf{b}_{-i}^{*}\right), \quad \forall i \in \mathbb{N}, \quad \forall b_{i} \geq 0
$$

We first establish the existence of the NE in our proposed relay auction as the following theorem.

TheOREM 1. There exists a bidding profile $B^{*}=\left\{b_{i}^{*}, \cdots, b_{N}^{*}\right\}$ to achieve Nash equilibrium in the relay auction.

Having established the existence of NE, we further investigate the uniqueness of this NE bidding profile in relay auction. To this end, we first construct $M R_{i}^{L_{1}}$ 's best response function (for fixed $\mathbf{b}_{-i}$ ) as:

$$
\beta_{i}\left(\mathbf{b}_{-i}\right)=\left\{b_{i} \mid b_{i}=\arg \max _{\widetilde{b_{i}} \geq 0} U_{i}^{L_{1}}\left(\widetilde{b_{i}} ; \mathbf{b}_{-i}\right)\right\}
$$

Then, we have the following theorem.

THEOREM 2. There exists a unique $N E B^{*}=\left\{b_{i}^{*}, \cdots, b_{N}^{*}\right\}$ in the relay auction.

Due to page limits, we omit the proof of the above theorems. We call our RA algorithm with linear payment function $f_{1}\left(b_{i}\right)=\frac{b_{i}}{A}$ and logarithmic payment function $f_{1}\left(b_{i}\right)=$ $\frac{\log \left(b_{i}+1\right)}{A}$ as RALiP and RALoP, respectively. Fig. 2 graphically depicts the existence and uniqueness of $\mathrm{NE}$ in case of $N=2$, where $a_{1}^{L_{2}}=a_{2}^{L_{2}}=10$, and $A=20$.
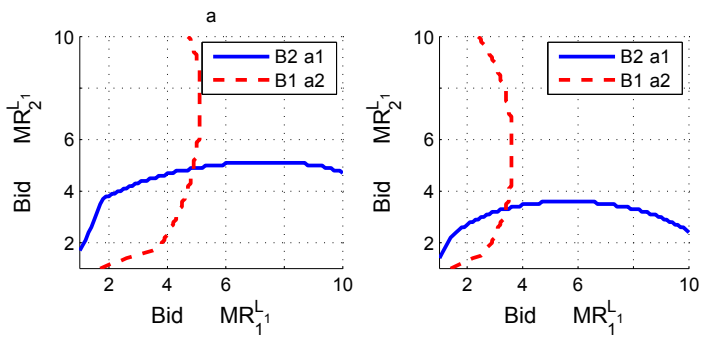

Figure 2: Best response functions: (a) RALiP; and (2) RALoP.

\subsection{Iterative Algorithm to Achieve NE}

To achieve NE in a practical distributed environment, one commonly used method is to allow users to iteratively update their strategies and bids according to best response functions[7]. However, it is difficult to get the close-form $\beta_{i}\left(\mathbf{b}_{-i}\right)$ in our model; and even if it exists, each $M R_{i}^{L_{1}}$ would have to know the bidding information $\mathbf{b}_{-i}$ of all other Level$1 \mathrm{MRs}$ so as to calculate its best response, which may not be feasible in practice unless the gateway provides MR such information.

Because the nonlinear constrained optimization problem defined by (21) can be converted to a convex optimization problem, and be efficiently solved by numerical methods, such as Lagrangian algorithm [4], or software solvers, such as MATLAB's Optimization Toolbox, and there exists a unique $\mathrm{NE}$ in the auction, we can design an asynchronous iterative bid updating algorithm to achieve NE based on each bidder's local information and the limited feedback received from the gateway. The key idea is to let the gateway help $M R_{i}^{L_{1}}$ to get other bidder's information by sending it to $M R_{i}^{L_{1}}$. Then with this information, each Level-1 MR can update its best response asynchronously and eventually converges to NE. For example, at time-slot $t$, the gateway receives bidding profile $\mathbf{B}$ from all bidders, it not only sends the allocation and payment results to all bidders, but also sends $\mathbf{b}_{-i}$ to $M R_{i}^{L_{1}}$. Then, $M R_{i}^{L_{1}}$ can calculate its best response $\beta_{i}\left(\mathbf{b}_{-i}\right)$ and send it to the gateway at time-slot $t+1$. We let each bidder update its bid asynchronously, e.g., $M R_{i}^{L_{1}}$ only updates at time $\{i, i+2 N, i+3 N, \cdots\}$. Then the NE can be achieved gradually.

\subsection{Implementation Considerations}

Current IEEE 802.16 protocol stack does not support auctionbased resource allocation. To realize our proposed auction framework and auction approaches in IEEE 802.16 systems, several implementation issues should be considered.

First, we consider to design a separate auction module that can be easily incorporated into current IEEE 802.16 architecture to realize auction-based resource allocation. As illustrated in Fig. 2, at each MR, the main protocol tasks are valuation and bidding process; while at the gateway, the tasks are time-slot allocation and payment calculation. Second, current IEEE 802.16-2004 standard defines 50 types of MAC management messages, which do not support auction. Thus, we propose to define five new MAC messages, i.e., Auction_Description, Auction_REG_REQ, Auction_REG ${ }_{-} R S P, B i d d i n g \_R E Q$, and Bidding_RST, to exchange auction information and results between the gateway and the MR. Specifically, as shown in Fig. 3, we assume that at the end of MR registration and MAC connection setup, the gateway sends (or broadcasts) an Auction_Description message to the newly registered MR, which includes the description of auction allocation and payment methods. Each Level-1 MR then registers to the auctioneer via Auction_REG_REQ, and the gateway responses with Auction_REG_RSP. With this procedure, the auctioneer can know the number of involved active bidders, and the number of served MCs in different MRs.

After auction registration and initialization, we can implement the bidding and allocation mechanisms during the process of bandwidth request. There are two main kinds of bandwidth request methods used in IEEE 802.16 system: contention-free polling and contention-based random access. 


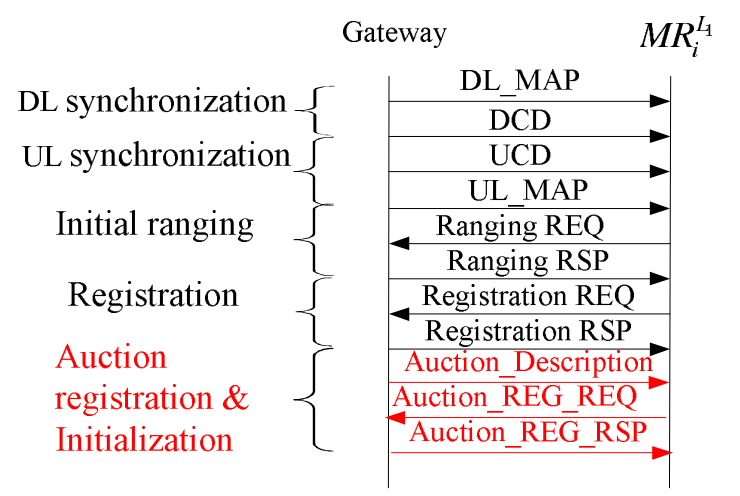

Figure 3: Auction registration and initialization.

Here, we propose to use the polling mechanism for the gateway to instruct MRs to request for bandwidth and submit bidding information. Specifically, as shown in Fig. 4, the bidder bids for resource via Bidding_REQ, while the auctioneer returns payment and allocation results via Bidding_RST, and then generate $D L \_M A P$ according to allocation results.

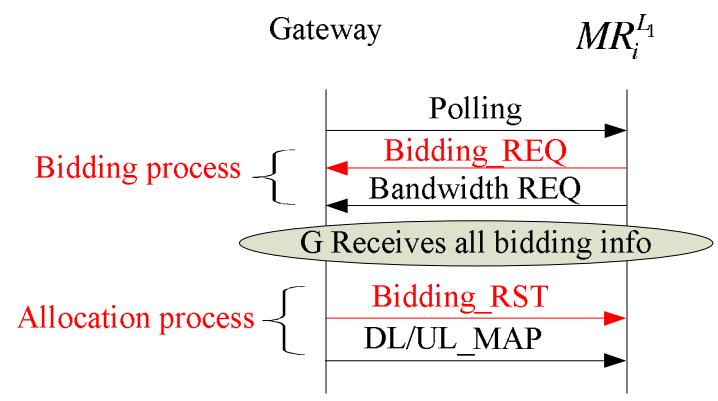

Figure 4: Auction process.

\section{PERFORMANCE RESULTS}

In this section, we provide simulations results to evaluate the performance of the auction algorithms.

\subsection{Simulation Configuration}

The simulation topology is shown in Fig. 1. Specifically, we assume each MR has 64 subcarriers with a total frequency band of $10 \mathrm{MHz}$. The frequency bands for adjacent MRs are non-coverlapping and frequency reuse is utilized. We further group 25 OFDM symbols into a slot, and let one DL-subframe have 10 time-slots. Thus, the DL-subframe length is $2 \mathrm{~ms}$. The wireless channel is modeled as six-path frequency-selective Rayleigh slow fading channel; each path is simulated by Clark's fading model and suffers from different fading with the maximum Doppler frequency of $30 \mathrm{~Hz}$. Because the coherence time $33.3 \mathrm{~ms}$ is much larger than the DL-subframe length, the channel condition can be regarded as static during the iterative bidding period to achieve NE. This is a realistic assumption in a WMN because the MRs are immobile.

We simulate CVCG, QVCG, RALiP and RALoP. We further assume with proper scheduling and queuing policies as well as adaptive modulation used in the MR, the packet error rate in $M R_{i}^{L_{1}}$ and $M R_{i}^{L_{2}}$ are all maintained at a constant value, and $P E R_{i}^{L_{1}}=1 \%$ and $P E R_{i}^{L_{2}}=1 \%$. In this setting, $N=2$ and there are $10 \mathrm{MCs}$ in each cell.

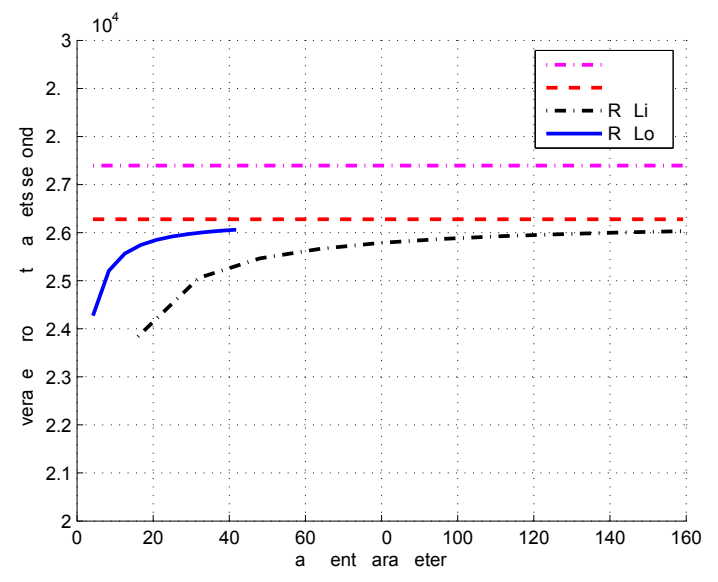

Figure 5: System average throughput vs. payment parameter.

Fig. 5 illustrates the system average throughput performance of the different four auction algorithms considered in our study. Here, to improve the readability of the figures, we only plot the curves of RALiP when the payment parameter $A$ is smaller than 40 . We can see that although VCGbased approaches generate higher throughput than those of the RA algorithms, the throughput of RALiP and RALoP achieve almost $95 \%$ of that for CVCG with the increase of payment parameter. This can be explained by the fact that the time-slots used by Level-1 MR to relay Level-2 MR's packets decrease if the payment parameter becomes higher. Because Level-1 MRs are close to the gateway and generally have better transmission conditions than those of Level-2 MRs, the overall system throughput increases when more resources are used for Level-1 MRs's traffic. On the other hand, RALoP is superior to RALiP. This is because the logarithmic payment function used by RALoP makes Level-1 MR pay less given the same bids, and thus, a larger portion of resource is allocated to $M R_{i}^{L_{1}}$

Now let us consider the fairness performance in terms of Jain fairness index [9] in Fig. 6. We can see that the Jain fairness indices for RALiP and RALoP are highly dependent on the payment parameter $A$. RALiP achieves its highest index value when $A=32$, while RALoP's fairness achieves its peak when $A=8$, and both are larger than that of CVCG and QVCG. Thus, the proposed RA algorithms can be used to strike a proper balance between efficiency and fairness, and achieve better fairness performance than VCG, while maintaining good efficiency performance.

To quantify the service dependability for Level-2 MRs in the system, we assume that each local connection generates 2500 packets per second in the downlink, and calculate the Level-2 MR's connection blocking probability (CBP) according to Engset formula [13]. The Level-2 MRs' average CBP performance is shown in Fig. 7. As discussed earlier, a higher payment parameter means that fewer time-slots can be used to relay Level-2 MRs'traffic. Thus, CBP values of RALiP and RALoP increase with $A$. When $A$ is relatively larger, such as $A>110$, the CBP values of RALiP and RALoP are higher than those of CVCG and QVCG. On the other hand, 


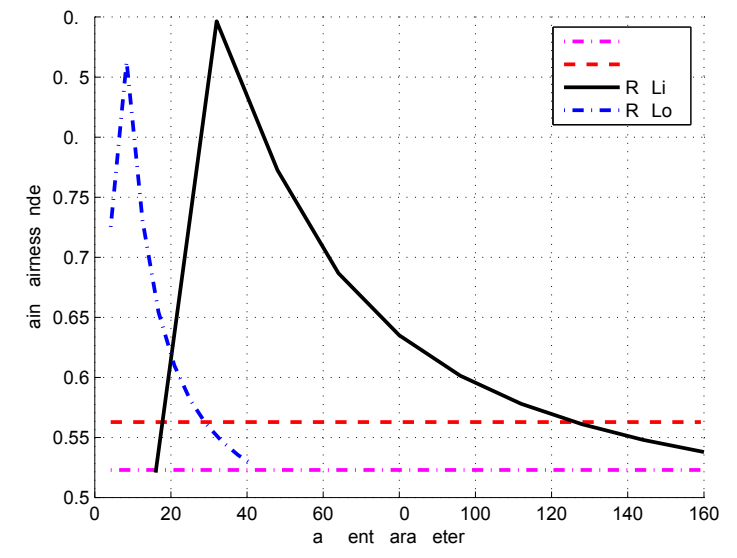

Figure 6: Jain fairness index vs. payment parameter.

when $A<62$ for RALiP and $A<15$ for RALoP, the RA algorithms have better CBP performance than VCG methods, demonstrating that they have a higher service dependability.

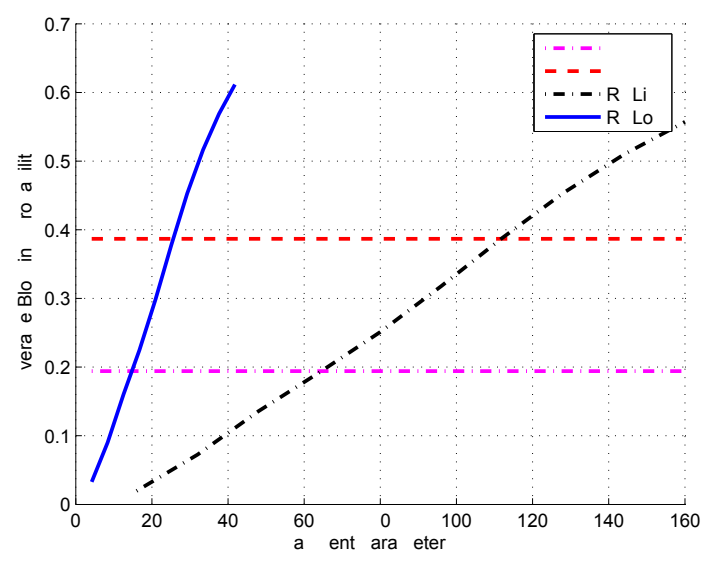

Figure 7: Level-2 CBP vs. payment parameter.

To investigate the scalability of the auction systems, we study the performance with four pairs of Level-1 and Level2 MRs, i.e., $N=4$. We first show the convergence of the bidding update algorithm. We let the true valuation parameters for these 4 Level-1 MRs be 10, 6, 4, and 8, respectively. In the simulation, the bidders first randomly submits their bids to the gateway, and then updates their best response iteratively. Fig. 8 and Fig. 9 indicate the convergence of both RALiP and RALoP, where the bids converge to NE eventually.

We then compare two traditional cooperative resource allocation methods in our non-cooperative environment:(1) proportional fairness (PF) [10], and (2) a global optimization method (GLB) which maximizes the following function:

$$
\begin{aligned}
\widetilde{T} & =\left\{\widetilde{T_{1}^{L_{1}}}, \widetilde{T_{1}^{L_{2}}}, \cdots, \widetilde{T_{N}^{L_{1}}}, \widetilde{T_{N}^{L_{2}}}\right\} \\
& =\arg \max _{\left\{T_{i}^{L_{1}}, T_{i}^{L_{2}}\right\}} \sum_{i} U_{i}^{L_{1}}\left(T_{i}^{L_{1}}\right)+U_{i}^{L_{2}}\left(T_{i}^{L_{2}}\right)
\end{aligned}
$$

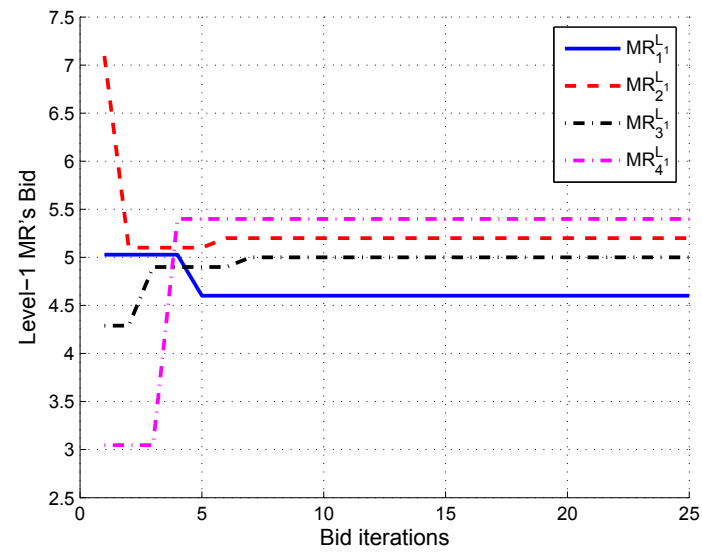

Figure 8: Convergence of bids to NE for RALiP.

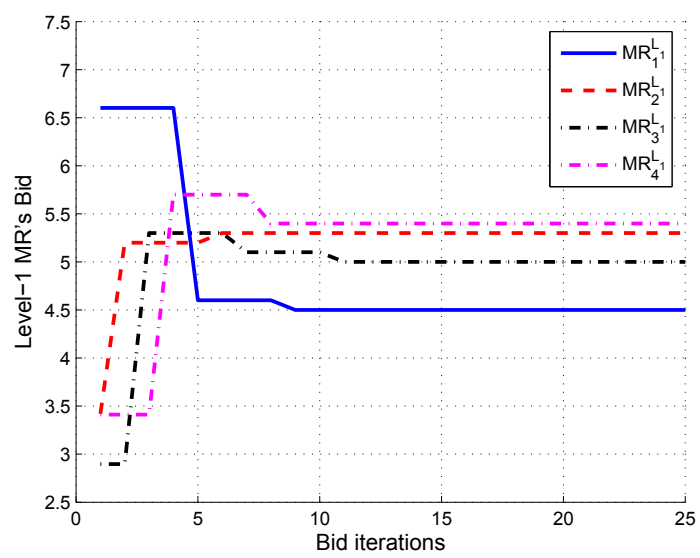

Figure 9: Convergence of bids to NE for RALoP.

subject to:

$$
\text { (I) } \sum_{i} T_{i}=T_{d} \quad(I I) T_{i}=T_{i}^{L_{1}}+T_{i}^{L_{2}}
$$

Notice that in a cooperative situation which is unrealistic in the operating environment considered in this paper, all MRs report their true valuations to the gateway, then the gateway allocate resource among different MRs based on these truthful information. However, in a non-cooperative environment, a selfish MR may report a bogus valuation parameter to gain a high payoff without regard to the overall system performance [11]. In our simulations, we assume $M R_{1}^{L_{1}}$ always report a higher valuation in terms of PF and GLB algorithms. As can be seen from Fig. 10, the system average throughput of PF and GLB are much lower than RA algorithms. Thus, it is quite clear that the traditional resource allocation algorithms are not suitable for a noncooperative WMN.

\section{CONCLUSIONS}

In this paper, we study the downlink bandwidth allocation problem in a non-cooperative IEEE 802.16 OFDM/TDMAbased hierarchical wireless mesh network in rural areas. We 


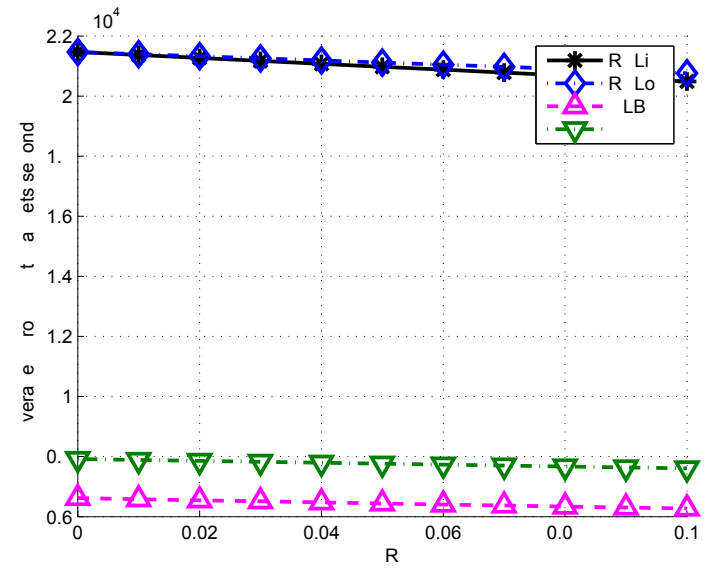

Figure 10: In noncooperative situation: System aggregate throughput vs. Level-2 PER.

design a novel auction framework to let rationally selfish mesh routers request resource actively and motivate them to forward other mesh routers' traffic. We then propose and analyze VCG-based auction approaches. To overcome the drawbacks of VCG-based auction algorithms, we further propose novel relay auction algorithms and analyze the bidding strategy using the solution concept of NE. After proving the existence and uniqueness of NE, an iterative bid updating algorithm is also designed to achieve NE in a practical distributed situation. Then we study the performance of these auction algorithms through simulations, which indicate that the proposed RA algorithms can achieve competitive performance in terms of resource allocation efficiency as the VCG approaches, while being able to adjust the resource ratio allocated to different levels of mesh routers, so as to strike a proper balance between efficiency and fairness. Furthermore, with a smaller payment parameter, the relay auction algorithms can also achieve a higher service dependability compared with the VCG algorithms. In the next step, we plan to extend our framework to a more general scenario with multiple gateways.

\section{REFERENCES}

[1] I. F. Akyildiz and X. Wang, "A Survey on Wireless Mesh Networks," IEEE Communications Mag., vol. 43, no. 9, pp. S23-S30, Sept. 2005.

[2] L. M. Ausubel and P. Milgrom, "The Lovely but Lonely Vickrey Auction," In Combinatorial Auctions, P. Cramton, Y. Shoham, and R. Steinberg (eds.), pp. 17-40, MIT Press, Cambridge, MA, 2006.

[3] H. T. Cheng and W. Zhuang, "Joint Power-Frequency-Time Resource Allocation in Clustered Wireless Mesh Networks," IEEE Network, pp. 45-51, Jan/Feb. 2008.

[4] E. K. P. Chong and S. H. Zak, An Introduction to Optimization, Third Edition, John Wiley and Sons, 2008.

[5] D. Fudenberg and J. Tirole, Game Theory, MIT Press, Cambridge, MA; London, England, 1991.

[6] G. He, S. Gault, M. Debbah, and E. Altman,"Distributed Power Allocation Game for
Uplink OFDM Systems," Proceedings of 6th International Symposium on Modeling and Optimization in Mobile, Ad Hoc, and Wireless Networks and Workshops (WiOPT 2008), pp. 515-521, Mar.-Apr. 2008.

[7] J. Huang, Z. Han, M. Chiang, and H. V. Poor, "Auction-Based Resource Allocation for Cooperative Communications," IEEE J. Select. Areas Communications, vol. 26, no. 7, pp.1226-1237, Sept. 2008.

[8] IEEE 802.16 Relay Task Group, 2008; http://www.ieee802.org/16/relay/

[9] R. Jain, D. Chiu and W. Hawe, "A Quantitative Measure of Fairness and Discrimination for Resource allocation in Shared Computer System," DEC Technical Report 301, 1984.

[10] F. Kelly, "Charging and Rate Control for Elastic Traffic," Eur. Trans. Telecommun., vol. 8, no. 1, pp. 33-37, Jan. 1997.

[11] Z. Kong, Y.-K. Kwok, and J. Wang, "On the Impact of Selfish Behaviors in Wireless Packet Scheduling," Proceedings of the IEEE International Conference on Communications (ICC 2008), pp. 3253-3257, May 2008.

[12] V. Krishna, Auction Theory, Academic Press, London, UK, 2002.

[13] M. Schwartz, Telecommunication Networks: Protocols, Modeling and Analysis, Addison- Wesley, Reading, MA. 1987.

[14] D. Niyato and E. Hossain, "A Radio Resource Management Framework for IEEE 802.16-Based OFDM TDD Wireless Mesh Networks," Proceedings of the 2006 IEEE International Conference on Communications (ICC 2006), pp. 3911-3916, June 2006.

[15] D. Niyato and E. Hossain, "Integration of IEEE 802.11 WLANs with IEEE 802.16-Based Multihop Infrastructure Mesh/Relay Networks: A Game-Theoretic Approach to Radio Resource Management,"IEEE Network, vol. 21, no. 3, pp. 6-14, May-June 2007.

[16] D. Niyato, E. Hossain, and V. K. Bhargava, "Scheduling and Admission Control in Power-Constrained OFDM Wireless Mesh Routers: Analysis and Optimization," IEEE Trans. Wireless Communications, vol. 6, no. 10, pp. 3738-3748, Oct. 2007.

[17] S. W. Peters and R. W. Heath, "The Future of WiMAX: Multihop Relaying with IEEE 802.16j," IEEE Communications Mag., vol. 47, no. 1, pp. 104-111, Jan. 2009.

[18] Y. Song, C. Zhang, and Y. Fang, "Joint Channel and Power Allocation in Wireless Mesh Networks: A Game Theoretical Perspective," IEEE J. Select. Areas Communications, vol. 26, no. 7, pp. 1149-1159, Sept. 2008.

[19] J. Sun, E. Modiano, and L. Zheng, "Wireless Channel Allocation Using an Auction Algorithm," IEEE J. Select. Areas Communications, vol. 24, no. 5, pp. 1085-1096, May 2006. 